\title{
Comparison of thyroid gland involvement in contrast enhanced computed tomography of the neck and histopathology and the necessity of thyroidectomy in patients with laryngeal and hypo pharyngeal cancer
}

\author{
Mohamed Abdul Kathar M.*, Rajiv Michael, Aparna Shyam
}

Department of ENT, CMC Vellore, Tamil Nadu, India

Received: 21 May 2019

Revised: 23 July 2019

Accepted: 30 July 2019

\section{*Correspondence:}

Dr. Mohamed Abdul Kathar M,

E-mail: drkadharshaji@gmail.com

Copyright: (c) the author(s), publisher and licensee Medip Academy. This is an open-access article distributed under the terms of the Creative Commons Attribution Non-Commercial License, which permits unrestricted non-commercial use, distribution, and reproduction in any medium, provided the original work is properly cited.

\section{ABSTRACT}

Background: The aim of this study is to assess the thyroid gland involvement in the preoperative contrast enhanced computed tomography (CECT) of neck with histopathology (HPE) in determining the indications for ipsilateral thyroidectomy in patients undergoing total laryngectomy in laryngeal and hypopharyngeal cancers.

Methods: Forty patients diagnosed clinically with stage 3 laryngeal and hypopharyngeal cancer were subjected to CECT of neck. Patients with cartilage erosion and extra laryngeal spread on CECT (T3, T4a) were routinely taken for total laryngectomy, partial pharyngectomy and hemi thyroidectomy. Specimens were sent for histopathological analysis and which was compared with preoperative CECT scan.

Results: Of the forty cases, only four patients had thyroid gland involvement in CECT scan and 2 of the 40 had thyroid gland involvement in histopathology. Of the four patients where the CT scan which showed infiltration of the thyroid gland, none had thyroid gland involvement in histopathology. In two patients where thyroid gland was involved in histopathology, CT scan failed to pick up the thyroid gland involvement. Those two patients with histologically proven thyroid gland involvement identify as transglottic malignancy with cartilage erosion and extra laryngeal spread.

Conclusions: Our study concludes that an ipsilateral hemi-thyroidectomy is probably still indicated in transglottic cancer with cartilage invasion and/or extra laryngeal spread.

Keywords: Thyroid gland, Thyroidectomy, Laryngeal and hypo pharyngeal cancers

\section{INTRODUCTION}

Laryngeal cancer is one of the common head and neck cancer, of which $95 \%$ are squamous cell carcinoma. The most commonest site being glottis $(51 \%)$, supraglottis $(33 \%)$ or subglottis $(2 \%)$ and $14 \%$ of the lesions are unable to be categorized. ${ }^{1}$ Laryngeal cancers can also invade the thyroid gland and the incidence ranges from 1$23 \%$ based on the histopathological specimens of total laryngopharyngectomy. ${ }^{2-5}$
The cause of thyroid gland invasion could either be due to the extra laryngeal spread by two ways: through cricothyroid membrane and laryngeal cartilage invasion. Since the thyroid gland is adjacent to larynx, the tumour infiltrates by contiguous spread or non-contiguously by lympho vascular invasion. Total laryngectomy is the treatment of choice for patients with $\mathrm{T} 3$ and T4a diseases in TNM staging. Tumour infiltrates the thyroid gland is considered as T4a. ${ }^{6}$ 
Ipsilateral hemi thyroidectomy or total thyroidectomy is routinely performed in all the patients undergoing total laryngectomy. But the role of performing thyroidectomy is controversial as thyroid gland invasion is very rare. Thyroidectomy results in long term morbidities like hypothyroidism and it has been reported in 23-63\%. The incidence increases to $70-91 \%$ when total laryngopharyngectomy with hemi thyroidectomy is followed by an adjuvant radiotherapy.,

Our objective of this study is to determine the prevalence of thyroid gland invasion and necessity of ipsilateral hemi thyroidectomy in patients undergoing total laryngopharyngectomy.

\section{METHODS}

This study was performed in the ENT outpatient department at the Christian medical college, Vellore, from April 2014 to December 2016. Institutional review board and ethical clearance was obtained for the conduct of this prospective study. Consecutive forty patients diagnosed to have stage 3 laryngeal cancer, based on clinical and endoscopic evaluation were recruited. Patients who experienced treatment failure with prior radiation therapy and underwent salvage laryngectomy were also included from this study.

All clinically diagnosed patients with stage 3 laryngeal cancers were subjected to contrast enhanced CT scan (from skull base to mediastinum) as a standard protocol of evaluation and staging. Patients with the biopsy proven laryngeal cancers (T3 and $\mathrm{T} 4 \mathrm{a}$ with cartilage erosion and/or extra laryngeal spread) were advised to undergo a total laryngectomy, partial pharyngectomy and ipsilateral hemi thyroidectomy and the surgical laryngectomy specimen was sent for histopathological examination. Thyroid gland infiltration findings in the histopathological results were compared with CT findings.

\section{Sample size calculation}

A sample of 40 patients will be required to obtain a $95 \%$ confidence interval of $\pm 10 \%$ around a positive predictive value of $88 \%$ in CT scan.

$$
n=\frac{4 p q}{d^{2}}
$$

$\mathrm{p}=$ positive predictive value $=88 \%$;

$\mathrm{d}=10 \%$

Where, $\mathrm{n}=$ sample size; $\mathrm{P}=$ sensitivity;

$\mathrm{Q}=100-P$

$\mathrm{d}=$ precision

\section{Statistical analysis}

From literature review data will be summarized with counts and percentages for categorical variables and mean and standard deviation for normally distributed continuous variables and median and range for nonnormally distributed 54 continuous variables. Chi-square test will be used for comparing the proportions between the groups. Diagnostics summary measures (sensitivity, specificity, predictive values) will be obtained with $95 \%$ confidence limits.

\section{RESULTS}

Of the forty study population, thirty nine (97\%) cases were male and one case $(3 \%)$ was female.

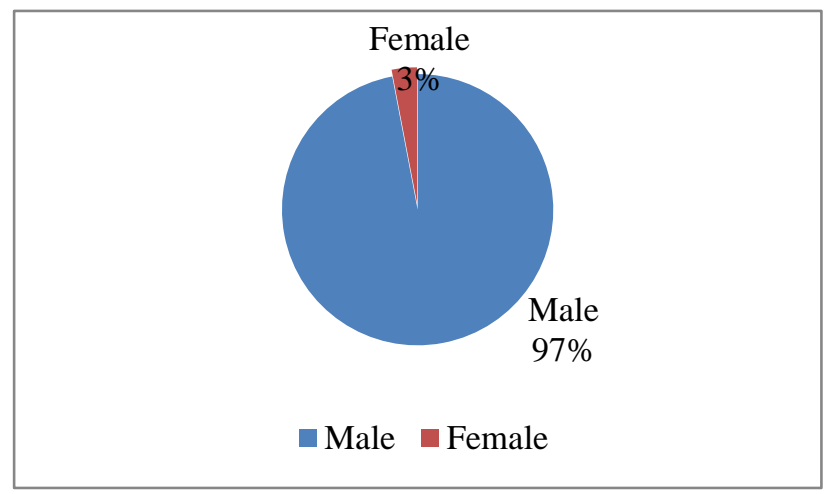

Figure 1: Sex ratio of the study population.

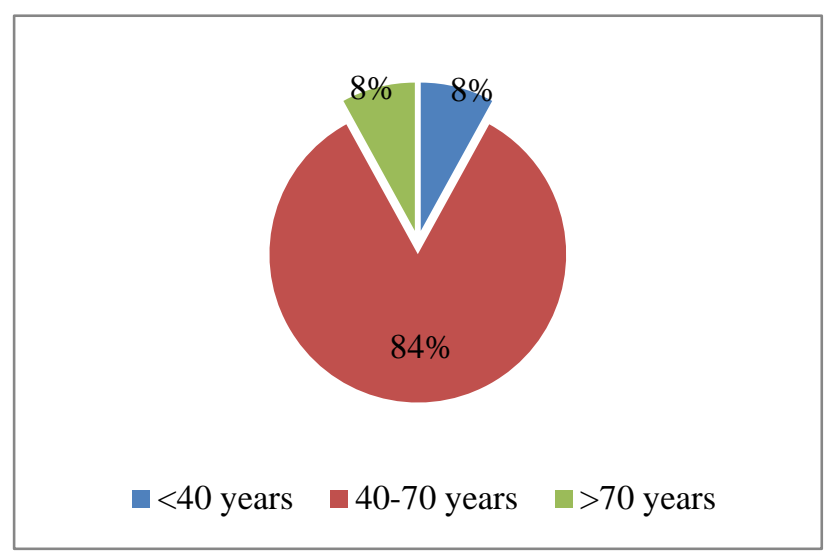

Figure 2: Age distribution pattern.

The age range was 35 years to 82 years with mean age of 74 years. Of the total 40 patients, $8 \%$ were found to be less than 40 years of age, $84 \%$ of patients were between 40-70 years of age and $8 \%$ were found to be more than 70 years of age.

Of the 40 cases, 19 were found to be glottis, 10 were hypopharyngeal carcinoma, 11 were supraglottis and 2 were found to be transglottic carcinoma. 


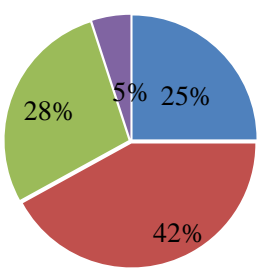

- Hypopharynx Glottic Carcinoma

- Supraglottic Carcinoma $\square$ Transglottic Carcinoma

Figure 3: Distribution of sub sites of laryngeal cancers.

Table 1: Comparison of thyroid gland involvement in CT neck with histopathology.

\begin{tabular}{|llll|}
\hline CT scan & HPE & & \\
\hline Yes & Yes & No & Total \\
\hline No & 0 & 4 & 4 \\
\hline Total & 2 & 34 & 36 \\
\hline
\end{tabular}

CT: Computed tomography; HPE: Histopathology.

Table 2: Diagnostic parameters with confidence interval of the study.

\begin{tabular}{|ll|}
\hline Parameters & $\begin{array}{l}\%(95 \% \text { confidence } \\
\text { limits) }\end{array}$ \\
\hline Positive predictive value & $0(0-60.2)$ \\
\hline Negative predictive value & $91.4(76.9-98.2)$ \\
\hline False positive & $0 \%$ \\
\hline Odds ratio & $0(0-12.9)$ \\
\hline False negative & $1.13(1-1.26)$ \\
\hline Sensitivity & $0(0-70.8)$ \\
\hline Specificity & $88.9(73.9-96.9)$ \\
\hline *. Confidence intervals not measurable.
\end{tabular}

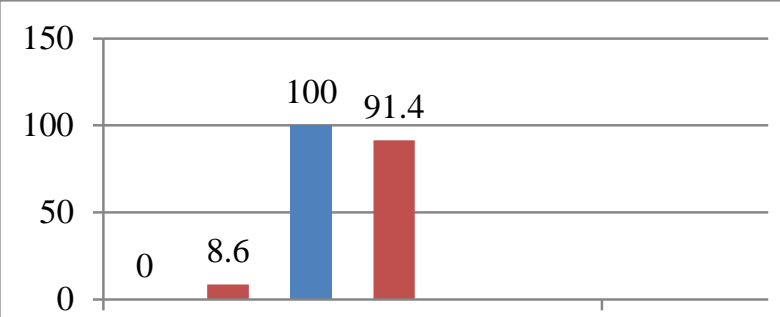

- Thyroid gland involvement in CT

- Thyroid gland involvement in Histopathology

Figure 4: Thyroid gland involvement in CT neck and histopathology.

In our study, out of the forty cases, only four patients had thyroid gland involvement on CT scan and 2 of the 40 had thyroid gland involvement on histopathology. Of the four patients where the CT scan showed infiltration of the thyroid gland, none had thyroid gland involvement in histopathology. In two patients where thyroid gland was involved in histopathology, CT scan failed to pick up the thyroid gland involvement. Those two patients with histologically proven thyroid gland involvement were diagnosed to have transglottic malignancy with cartilage erosion and extra laryngeal spread.

\section{DISCUSSION}

Thyroid gland infiltration by laryngeal cancer can be due to either direct extension or lympho vascular invasion. ${ }^{8}$ The prevalence of thyroid gland involvement reported in the literature is only $13 \%$. Hence thyroidectomy is performed unnecessarily. Extra laryngeal extension, which include the cricopharyngeus and cricothyroid muscles along with the surrounding fascia are the first to be involved when the disease spreads laterally outside the skeletal framework of larynx. Mere involvement of anterior commissure cannot be considered as an indication for thyroidectomy as vertical distance between the anterior commissure and the isthmus is about $2-3 \mathrm{~cm}$.

Computed tomography of the neck is the essential modality of choice to identify the need for thyroidectomy. Review of preoperative CECT scans revealed that radiologic evidence for thyroid cartilage invasion had similar proportions in both patients with and without thyroid gland involvement. There is no contribution of MRI to thyroid gland staging could be found in the literature, despite the very precise soft tissue imaging provided by this modality.

In a study done by Hilly et al, thyroidectomy along with laryngectomy was performed in 52 patients. Thyroid gland involvement was evident in $21 \%$ (11 patients) of the $88 \%$ who underwent total laryngectomy with thyroidectomy. ${ }^{10}$ In his study, CT detected thyroid gland involvement in 1 of 11 patients, whereas the surgical specimen showed thyroid involvement in 3 of the 11 cases. ${ }^{11}$

In our study, out of the forty cases, only four patients had thyroid gland involvement on CT scan and 2 of the 40 had thyroid gland involvement in histopathology. Of the four patients where the CT scan showed infiltration of the thyroid gland, none had thyroid gland involvement in histopathology. In two patients where thyroid gland was involved in histopathology, CT scan failed to pick up the thyroid gland involvement. Those two patients with histologically proven thyroid gland involvement were diagnosed to have transglottic malignancy with cartilage erosion and extra laryngeal spread.

Transglottic tumours have a higher incidence of extra laryngeal spread as pointed in a study by Nayak et al. ${ }^{12}$ He emphasised that that transglottic growths and extra laryngeal soft tissue infiltration to be statistically 
significant factors that would warrant an ipsilateral hemi thyroidectomy during total laryngectomy.

Numerous studies have indicated that transglottic tumours are large volume lesions that tend to spread laterally and there is an increased risk of thyroid gland invasion for these tumours.

\section{CONCLUSION}

In our study, we looked at the indications for ipsilateral thyroidectomy in patients undergoing total laryngectomy. Contrast enhanced CT scan suggested thyroid gland infiltration in four out of the forty cases. Histopathology was negative in all these four cases. Two other cases showed thyroid gland infiltration in histopathology but not in CECT scan. These two cases with thyroid gland involvement in histopathology were both transglottic tumours with extra laryngeal spread. Hence an ipsilateral hemi thyroidectomy is probably still indicated in transglottic cancer with cartilage invasion and/or extra laryngeal spread.

\section{ACKNOWLEDGEMENTS}

It is a pleasant task to express my sincere thanks to all those who contributed in many ways to the successful completion of this study.

\section{Funding: No funding sources}

Conflict of interest: None declared

Ethical approval: The study was approved by the Institutional Ethics Committee

\section{REFERENCES}

1. American Cancer Society. Cancer facts and figures, 2010. Atlanta, GA: American Cancer Society; 2010.

2. Sparano A, Chernock R, Laccourreye O, Weinstein $\mathrm{G}$, Feldman M. Predictors of thyroid gland invasion in glottic squamous cell carcinoma. Laryngoscope. 2005; 115:1247-50.

3. Kim JW, Han GS, Byun SS, Lee DY, Cho BH, Kim YM. Management of thyroid gland invasion in laryngopharyngeal cancer. Auris Nasus Larynx. 2008;35:564-7.
4. Biel MA, Maisel RH. Indications for performing hemi thyroidectomy for tumors requiring total laryngectomy. Am J Surg. 1985;150:435-9.

5. Dadas B, Uslu B, Cakir B, Ozdogan HC, Calis AB, Turgut S. Intraoperative management of the thyroid gland in laryngeal cancer surgery. J Otolaryngol. 2001;30:179-83.

6. Psychogios $\mathrm{G}$, Waldfahrer $\mathrm{F}$, Bozzato $\mathrm{A}$, Iro $\mathrm{H}$. Evaluation of the revised TNM classification in advanced laryngeal cancer. Eur Arch Otorhinolaryngol. 2010;267:117-21.

7. Biel MA, Maisel RH. Indications for performing hemi thyroidectomy for tumours requiring total laryngectomy. Am J Surg. 1985;150:435-9.

8. Brennan JA, Meyers AD, Jafek BW. The intraoperative management of the thyroid gland during laryngectomy. Laryngoscope. 1991;101:92934.

9. Keberle M, Kenn W, Hahn D. Current concepts in imaging of laryngeal and hypopharyngeal cancer. Europ Radiol. 2002;12:1672-83.

10. Ljumanovic R, Langendijk JA, Hoekstra OS, Leemans CR, Castelijns JA. Distant metastases in Head and neck carcinoma: identification of prognostic groups with MR imaging. Eur J Radiol. 2006;60(1):58-66.

11. Hilly O, Raz R, Vaisbuch Y, Strenov Y, Segal K, Koren R, et al. Thyroid gland involvement in advanced laryngeal cancer: association with clinical and pathologic characteristics. Head Neck. 2012;34(11):1586-90.

12. Nayak SP, Singh V, Dam A, Bhowmik A, Jadhav TS, Ashraf M, et al. Mechanism of thyroid gland invasion in laryngeal cancer and indications for thyroidectomy. Indian J Otolaryngol Head Neck Surg. 2013;65(1):69-73.

Cite this article as: Kathar MMA, Michael R, Shyam A. Comparison of thyroid gland involvement in contrast enhanced computed tomography of the neck and histopathology and the necessity of thyroidectomy in patients with laryngeal and hypo pharyngeal cancer. Int J Otorhinolaryngol Head Neck Surg 2019;5:1335-8. 